\title{
Aplicativos de tecnologia móvel desenvolvidos na geração de informações relacionados a atenção primária a saúde: análise da contribuição brasileira
}

\author{
Mobile technology applications developed in the generation of information related to \\ primary health care: analysis of the Brazilian contribution
}

Aplicaciones de tecnología móvil desarrolladas en la generación de información relacionada con la atención primaria de salud: análisis de la contribución brasileña

José de Ribamar Ross ${ }^{1 *}$, Lara Ramayanne da Silva Rodrigues ${ }^{1}$, Gabriel Rodrigues Côra', Lívia Maria Nunes de Almeida ${ }^{2}$, Kelvya Fernanda Almeida Lago Lopes ${ }^{1}$, Conceição de Maria Lima Soares Ximenes $^{3}$, Irene Sousa da Silva ${ }^{1}$, Maria de Jesus Lopes Mousinho Neiva ${ }^{1}$, Galbia Nelma Silva Rodrigues Santos ${ }^{4}$, Nytale Lindsay Cardoso Portela ${ }^{5}$.

\section{RESUMO}

Objetivo: Destacar os aplicativos desenvolvidos para utilização na Atenção Primaria à Saúde. Métodos: Revisão integrativa de 19 artigos indexados na base de dados eletrônica Biblioteca Virtual em Saúde (BVS), publicados nas línguas inglesa, espanhola e portuguesa, no período de 2010 e 2020, que abordavam diferentes ferramentas para aplicação na área da saúde. Resultados: Destacaram-se os artigos publicados em países da América e Europa, na língua inglesa, no período de 2015 a 2019 e abordavam os seguintes temas: condições crônicas, nutrição/dietoterapia/educação física, saúde coletiva, saúde mental/psiquiatria, triagem auditiva e doenças oculares. A maioria dos artigos foram avaliados após à aplicação na comunidade e obtiveram aceitação e resultados promissores. Considerações finais: Os aplicativos de tecnologia móvel desenvolvidos na geração de informações relacionados à atenção primária a saúde concentra-se na área de atenção as condições crônicas principalmente para orientar profissionais e paciente sobre rastreamento, diagnóstico e tratamento. A participação brasileira na produção e publicação demonstradas nesta tecnologia foi discreta.

Palavras-chave: Saúde pública, Atenção primária à saúde, Tecnologia da informação, Aplicativos móveis.

\begin{abstract}
Objective: To highlight the applications developed for use in Primary Health Care. Methods: Integrative review of 19 articles indexed in the electronic database Virtual Health Library (VHL), published in English, Spanish and Portuguese, in the period 2010 and 2020, which addressed different tools for application in the health area. Results: The articles published in American and European countries, in English, from 2015 to 2019, were highlighted and addressed the following topics: chronic conditions, nutrition/diet therapy/physical education, public health, mental health/psychiatry, screening hearing and eye diseases. Most articles were evaluated after application in the community and obtained acceptance and promising results. Final considerations: Mobile technology applications developed in the generation of information related to primary health care are focused on the area of care for chronic conditions mainly to guide professionals and patients on screening, diagnosis and treatment. The Brazilian participation in the production and publication demonstrated in this technology was discreet.
\end{abstract}

Keywords: Public health, Primary health care, Information technology, Mobile applications.

\footnotetext{
1 Universidade Estadual do Maranhão (UEMA), Caxias - MA. *E-mail: joseross@professor.uema.br.

2 Universidade Federal do Piauí (UFPI), Floriano - PI.

${ }^{3}$ Centro Universitário de Ciências e Tecnologia do Maranhão (UniFacema), Caxias - MA.

${ }^{4}$ Hospital Universitário Presidente Dutra (HUUFMA), São Luís - MA.

${ }^{5}$ Fundação Oswaldo Cruz (Fiocruz), Rio de Janeiro - RJ.
} 


\section{RESUMEN}

Objetivo: Destacar las aplicaciones desarrolladas para su uso en Atención Primaria de Salud. Métodos: Revisión integradora de 19 artículos indexados en la base de datos electrónica Biblioteca Virtual en Salud (BVS), publicados en inglés, español y portugués, en el período 2010 y 2020, que abordaron diferentes herramientas de aplicación en el área de la salud. Resultados: Los artículos publicados en los países de América y Europa, en inglés, de 2015 a 2019, se destacaron y abordaron los siguientes temas: enfermedades crónicas, nutrición / dietoterapia / educación física, salud pública, salud mental / psiquiatría, cribado auditivo y ocular. enfermedades. La mayoría de los artículos fueron evaluados después de su aplicación en la comunidad y obtuvieron aceptación y resultados prometedores. Consideraciones finales: Las aplicaciones de tecnología móvil desarrolladas en la generación de información relacionada con la atención primaria de salud están enfocadas en el área de atención a las condiciones crónicas principalmente para orientar a los profesionales y pacientes en el cribado, diagnóstico y tratamiento. La participación brasileña en la producción y publicación demostrada en esta tecnología fue discreta.

Palabras clave: Salud pública, Atención primaria de salud, Tecnología de la información, Aplicaciones móviles.

\section{INTRODUÇÃO}

De acordo com Mendes EV, et al. (2019) a Atenção Primária à Saúde (APS) é a porta de entrada no sistema de saúde. Considerando as modificações epidemiológicas ocorridas no país e a relevância das condições crônica no cenário atual, observa-se pouca disponibilidade de aplicativos de tecnologia móvel para usuários e profissionais de saúde da APS úteis nas ações de cuidado. Assim, com o objetivo de contribuir nas ações de rastreamento dessas condições crônicas e suporte para o seu tratamento faz-se necessário investigar evidências relacionadas ao desenvolvimento de aplicativos de tecnologia móvel que podem ser utilizados por usuários da atenção primária a saúde.

Atualmente, as funções dos telefones celulares atingiram um nível de complexidade tão alto que permitem o acesso a informações e à aplicativos desses dispositivos a qualquer hora e em qualquer lugar, e agora são chamados de smartphones. Após a revolução desencadeada pela Internet e redes sociais, a popularidade desses dispositivos constitui a revolução tecnológica mais influente dos últimos tempos (TIBES CMS, et al., 2014).

A dinâmica social atual da sociedade e caracterizada principalmente pelo alto potencial de comunicação. A inserção do ambiente virtual no cotidiano dos indivíduos e um fato mundial, que se deve grande parte a globalização. Dentro deste cenário, observa-se um avanço frequente das Tecnologias da Informação e Comunicação (TICs), que vem se destacando em várias áreas de conhecimento (DA ROCHA FS, et al., 2017).

As TICs são tecnologias de comunicação que facilitam a transmissão de informações através de métodos digitais, como computadores e redes sem fio. Essas TICs têm sido utilizadas mundialmente tanto em contextos pessoais, como também na área educacional, empresarial e atualmente, essas tecnologias têm sido implantadas no âmbito dos serviços de saúde (SILVA AB, 2019).

As TICs para o setor saúde detém de uma grande variedade de ferramentas que podem apoiar a estruturação e organização de dados e informações, além de realizar armazenamento, processamento, acesso e compartilhamento, a manipulação das TIC pode ser realizada por profissionais que estão envolvidos na assistência ou o próprio paciente. Este tipo de tecnologia é considerado um recurso global que conecta múltiplos computadores para criar uma rede de informação e permite a colaboração com o desenvolvimento e aprimoramento da profissão de saúde. Além da capacidade de disseminar, disseminar e atualizar conhecimentos na área da saúde, as TIC também podem auxiliar na tomada de decisão clínica dos profissionais para ajudar a formular diagnósticos confiáveis e orientações ou condutas de tratamento qualificados para pacientes (ROBERTS S, et al., 2017).

Dentro da área da saúde, na enfermagem, por exemplo é crescente o desenvolvimento e utilização das TICs, visto que é um mecanismo didático contemporâneo, que tem como um dos pilares a automatização de processos, e tem como uma de suas principais vantagens, a organização e a segurança no processo (PEREIRA FGF, et al., 2016). 
No momento, podemos ver a proliferação de tecnologia e aplicativos móveis (m-health), e eles estão colaborando para construir um novo modelo de assistência na saúde, onde as informações sobre a saúde das pessoas se tornam mais acessíveis. Vários estudos têm mostrado que tais aplicativos, incluindo as informações que geram, podem ser utilizadas para otimizar resultados e reduzir riscos à saúde, bem como para compreender os determinantes da promoção da saúde e doença (MARCANO BJS, et al., 2015).

O m-health (saúde móvel) discorre inicialmente os problemas que ocorrem durante os primeiros atendimentos na Atenção Básica, incluindo o aumento do número de doenças crônicas relacionadas ao estilo de vida. Além desses fatores, contribui também para a redução do alto custo dos serviços nacionais de saúde existentes, além de expor o paciente e sua família sobre a importância do cuidado de si mesmo, promovendo uma maior atenção integral a saúde. O m-health como uso criativo de novas informações de saúde móvel e tecnologia de detecção, tem o potencial de reduzir os custos relacionados ao cuidado e melhorar a pesquisa e os resultados de saúde. Essas tecnologias podem apoiar o monitoramento contínuo nos níveis individual e populacional, encorajar comportamentos saudáveis para prevenir ou reduzir problemas de saúde, apoiar o autogerenciamento de doenças crônicas, melhorar o conhecimento dos provedores, reduzir o número de consultas médicas e fornecer intervenções sob demanda uma forma que antes era inimaginável (SILVA BMC, et al., 2015).

Hoje, existe uma necessidade de repensar as inúmeras possibilidades da tecnologia móvel nas ações de saúde, pois é reconhecido que a aplicabilidade da tecnologia digital de informação e comunicação não está apenas na saúde, mas em diferentes áreas do conhecimento. Este tópico é tão importante que a Organização Mundial da Saúde criou o Global Observatory for eHealth (Observatório Mundial de Saúde Eletrônica) para promover, divulgar e gerar dados que ajudem a saúde e fornecer informações importantes para apoiar o governo em sua futura decisão de política de uso nesta tecnologia (GAMA LN e TAVARES CMM, 2019).

A principal vantagem dos dispositivos moveis em relação aos meios mais tradicionais, como os computadores, tem sido o fato de que podem ser levados com facilidade para qualquer lugar e geralmente estão conectados à internet. Diversos estudos atuais se voltaram a analisar a utilização das tecnologias na saúde. Ao analisar a utilização de tecnologias de saúde baseadas em smartphones, constatou-se que muitos aplicativos para dispositivos móveis vêm sendo desenvolvidos e utilizados no em áreas educacionais, como o ensino de profissionais da saúde e também aplicativos destinados a utilização dos pacientes, como aplicativos de autogerenciamento de doenças e monitoramento remoto de pacientes. Quando se analisa a utilização dessas ferramentas no Brasil, evidencia-se que as ferramentas disponibilizadas atualmente, em sua maioria, são destinadas ao apoio aos profissionais de saúde (SILVA AB, 2019).

Vale destacar também que a pesquisa na área da saúde também pode se beneficiar da tecnologia móvel, pois esta tecnologia permite o registro de uma grande quantidade de informações geradas por essas pesquisas, o que é muito útil para otimizar a fase de organização e processamento da informação, bem como para gerenciar a segurança do fluxo de dados e agilidade, disponibilizando-os rapidamente sendo interessante sua utilização no desenvolvimento da pesquisa e a análise dos resultados (PEREIRA IM, et al., 2017).

$\mathrm{Na}$ literatura internacional, os aplicativos de saúde registrados são categorizados de acordo com a aplicação para os profissionais de saúde. O foco está em diagnóstico médico assistido, referência de medicamentos, pesquisa de literatura, comunicação clínica, treinamento médico e de enfermagem e aplicativos para pacientes focado em gestão de doenças crônicas e suas aplicações, também são encontrados aplicativos para estudantes de medicina e enfermagem (MOSA AS, et al., 2012).

Nos últimos anos, o uso de inovações tecnológicas em saúde tem conduzido pesquisas sob diferentes perspectivas, resultando em aplicações principalmente para monitoramento de pacientes, com foco em métodos multidisciplinares, suporte remoto ao paciente e apoio na tomada de decisão em equipe. Dentre eles, a média a alta complexidade costuma ser o principal espaço de aplicação dessas ferramentas (TIBES CMS, et al., 2014; BARRA CCD, et al., 2017).

O objetivo deste artigo foi destacar os aplicativos desenvolvidos para utilização na Atenção Primaria à Saúde. 


\section{MÉTODOS}

Este estudo trata-se de uma revisão integrativa de caráter exploratório descritivo. A coleta de dados foi realizada em janeiro de 2021 e elegeram-se artigos indexados na base de dados eletrônica Biblioteca Virtual em Saúde (BVS). Foram utilizados os descritores: Aplicativos móveis e Atenção Primaria à Saúde. Foram incluídos todos os artigos publicados nos últimos 10 anos, a fim de não excluir nenhum aplicativo criado, considerando a revolução 4.0 ocorrida neste intervalo de tempo; em língua portuguesa, inglesa e espanhola, que abordassem a criação e avaliação de aplicativos para dispositivos móveis. Foram excluídos: artigos de revisão, artigos que abordavam aplicativos destinados a outros níveis de atenção (atenção secundária e terciaria). Na busca, foram utilizados os operadores booleanos: AND, OR e NOT permitindo filtrar melhor a busca junto a base (Figura 1).

Figura 1 - Diagrama de fluxo do processo de seleção das produções científicas.

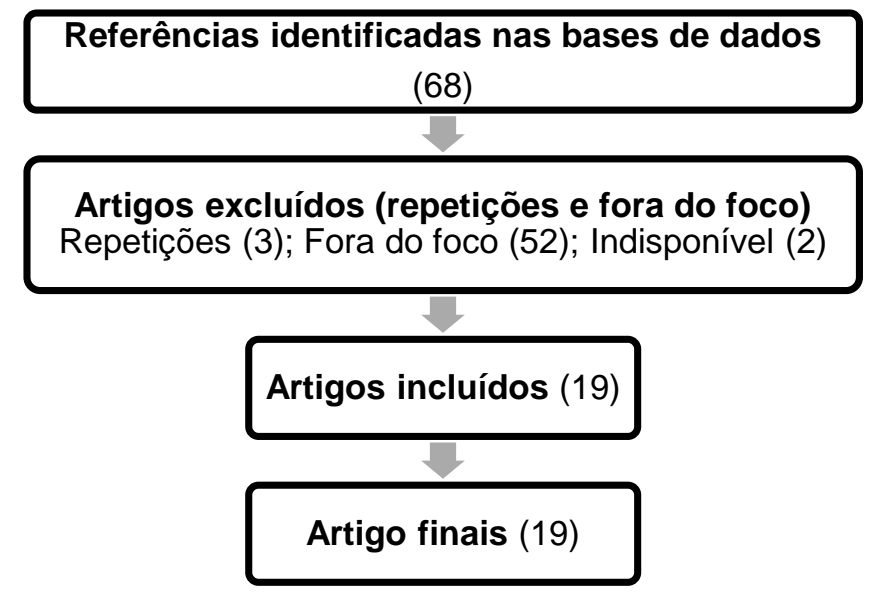

Fonte: Ross JR, et al., 2022.

A estratégia de busca utilizou os descritores "Aplicativos móveis e Atenção Primaria à Saúde" combinados com os operadores boleanos obteve-se um resultado de 102 artigos no formato de textos completos. Dentre esses, foram aplicados os filtros: publicados nos últimos 10 anos, resultando em um total de 68 artigos.

Foi realizada a leitura criteriosa dos artigos onde após leitura atenciosa obteve-se uma seleção de 23 artigos, sendo excluídos artigos de revisão, artigos repetidos ou que fugiam do foco sendo excluídos 4 artigos. Ao final foram selecionados 19 artigos. Após etapas de seleção os artigos foram analisados de acordo com variáveis descritivas e os dados foram agrupados em tabelas.

\section{RESULTADOS}

Com relação aos países de origem, encontrou-se: 7 artigos dos Estados Unidos, 3 brasileiros, 1 canadense, 3 da Espanha, 1 da Bélgica, 1 do Reino Unido, 1 da África do Sul e 2 australianos (Quadro 1).

Ao analisar a filiação dos principais autores de cada estudo, constatou-se que em 14 artigos $(73,7 \%)$ os autores principais eram profissionais da saúde, sendo $12(63,2 \%)$ da área médica e $4(10,5 \%)$ da Nutrição. Em 4 artigos (10,5\%), os autores principais eram profissionais da ciência digital e em 1 artigo (5,3\%), o autor era da área de humanas (Geografia).

Em relação a língua de publicação dos artigos, a maioria foi em inglês $(84,2 \%)$, seguida do português e espanhol (15,8\%). Quanto ao tipo de estudo, 47,3\% corresponderam a ensaios clínicos randomizados, 15,8\% a estudos de caso, 15,8\% a estudos descritivos exploratórios, 10,5\% a estudos de intervenção clínica, 5,3\% a estudo de precisão diagnóstica e 5,3\% a relato de caso.

A maioria $(36,8 \%)$ dos artigos foram publicados no ano de 2018, $6(31,6 \%)$ artigos publicados em 2017, 3 $(15,8 \%)$ em 2019, 2 (10,5\%) em 2015 e 1 (5,3\%) publicados no ano de 2016 (Quadro 1). 
Quadro 1 - Distribuição de artigos segundo aplicativo, país de origem e área.

\begin{tabular}{|c|c|c|c|c|}
\hline $\mathbf{N}$ & Autores (ANO) & País & Aplicativo & Área \\
\hline 1 & PACHECO KCF, et al., 2018 & BR & Conect Agent & Doenças transmissíveis \\
\hline 2 & POSSEMATO K, et al., 2017 & US & PTSD Coach & Saúde mental \\
\hline 3 & LOUW C, et al., 2017 & ZA & Hear Screen & Triagem auditiva \\
\hline 4 & $\begin{array}{c}\text { RECIO-RODRIGUEZ JI, et al., } \\
2018\end{array}$ & ES & Evident II & Nutrição \\
\hline 5 & AHERN DK, et al., 2016 & US & Lung Age & Triagem pulmonar \\
\hline 6 & GRANADO-FONT E, et al., & ES & Sem nome & Alimentação e Atividades \\
físicas
\end{tabular}

Legenda: AR: Argentina, AU: Austrália, BE: Bélgica, BR: Brasil, CA: Canadá, ES: Espanha, US: Estados Unidos, ZA: África do Sul. Fonte: Ross JR, et al., 2022.

Os estudos variaram quanto a área temática dos conteúdos assim, destacaram-se: Condições crônicas 9 (47,4\%); Nutrição/dietoterapia/educação física 4 (21,1\%); Saúde coletiva 1 (5,3\%); Saúde mental/psiquiatria 3 (15,8\%); Triagem auditiva 1 (5,3\%) e; Doenças oculares 1 (5,3\%). Referente aos que trabalharam condições crônicas destacaram-se os relacionados a câncer, doenças pulmonares, obesidade, tabagismo e gestação (Quadro 1).

Com relação à avaliação das intervenções, observou-se que a maioria tem conteúdo instrutivo na finalidade ao qual foi destinada, oferecendo suporte às atividades de diagnósticos, rastreamento e gerenciamentos. Quatro artigos ainda não apresentavam avaliações quanto ao desempenho das intervenções.

Os aplicativos foram testados e avaliados de acordo com o seu público-alvo e apresentaram em sua maioria resultados satisfatórios, alta especificidade e sensibilidade, segundo Louw C, et al. (2016), viabilidade de implantação, conforme afirmou Tsoh JY, et al. (2018) e resultados superiores quando utilizados em associação com os procedimentos padrão que não utilizavam intervenções digitais, como constatou RecioRodriguez JI (2018), em seu estudo sobre o aplicativo Evident II. Dentre esses artigos, um apresentou bom desempenho, porém, de acordo com a pesquisa, necessita de mais estudos para saber se a ferramenta é capaz de reduzir a mortalidade materna, que é o foco da intervenção (SILVA AB, 2019).

Os aplicativos abordados nos artigos utilizavam recursos educativos como vídeos, mensagem informativas e textos explicativos. Além disso, os aplicativos voltados para rastreamento, autogerenciamento e triagem, além de recursos educativos apresentavam também questionários rápidos.

A respeito das melhorias e possíveis impasses que essas ferramentas pudessem apresentar, observouse uma grande variedade de recomendações. Sete artigos não fizeram observações acerca de alterações a serem realizadas nos aplicativos. Segundo Posemato K, et al. (2016), Maulik PK, et al. (2017), Silva AB (2019) e Proesmans T, et al. (2019), suas aplicações necessitam de testes em outras áreas e de mais estudos que reafirmem a sua eficácia. 


\section{DISCUSSÃO}

Acredita-se que os recursos de tecnologia da informação e comunicação, principalmente através de aplicativos móveis, que permitem o acesso à informação a qualquer momento, são ferramentas em potencial para melhoria do acompanhamento, gestão, autocuidado e outras funcionalidades dentro dos serviços de saúde. Além disso, as tecnologias de saúde móvel são promissoras para melhorar o ambiente de saúde, uma vez que trazem resultados benéficos também para os pacientes, como maior agilidade nos resultados, autonomia no autocuidado, e menor custo nos tratamentos (DAHNE J, et al., 2017).

Um dos principais benefícios dos recursos tecnológicos é que eles permitem o acesso em tempo real e de forma remota às informações, ou seja, contribuem para a solução de problemas e atendimento de necessidades de saúde em diferentes áreas geográficas. Vemos, portanto, que o uso da tecnologia no âmbito do cuidado em saúde, facilita o trabalho, na medida em que agiliza, traz maior precisão e rapidez nas ações, o que propicia um maior tempo para a equipe se dedicar ao cuidado. Sendo assim, pode-se afirmar que a utilização desses recursos, é capaz de melhorar a qualidade da assistência (DAHNE J, et al., 2017).

Um estudo realizado na atenção primária à saúde na Etiópia comparou a precisão dos dados do paciente coletados por meio de formulários eletrônicos em smartphones com a precisão dos dados do paciente coletados em formulários de papel apontando que, em comparação com os formulários de papel, o uso de planilhas bem elaboradas tem resultados significativos melhorando os registros de integridade de dados em $8 \%$ concluindo - se que, por meio de treinamento e supervisão, os profissionais de saúde são capazes de usar planilhas de forma adequada e precisa para avaliação do paciente e coleta de dados de rotina (MEDHANYIE AA, 2017).

No Brasil, o e-SUS é referência em meio eletrônico utilizado na atenção básica do Sistema Único de Saúde (SUS), foi lançado com o objetivo de desenvolver, reorganizar e integrar sistemas de informação de forma que permitam a transmissão simultânea de informações entre todas as áreas de assistência do governo, visando reduzir riscos e melhorar os resultados de saúde do paciente, que são os requisitos enfrentados pelo sistema de saúde global (BRASIL, 2014).

Dentre as ferramentas tecnológicas que podem ser utilizadas dentro da atenção à saúde, pode-se destacar os aplicativos móveis. Eles estão relacionados com a quebra da limitação da mobilidade, visto que acompanham o usuário onde quer que esteja e tem a capacidade de trazer pessoalidade aos seus usuários, uma vez que podem ser utilizados como aparelho pessoal (OLIVEIRA ARF e ALENCAR MSM, 2017).

A tecnologia móvel permite a configuração de um novo cenário no qual são estimulados hábitos saudáveis, o manejo adequado de doenças crônicas e diversos outros aspectos, pois pode criar condições para a avaliação contínua dos parâmetros de saúde. Porém, para que os usuários entendam seu uso, além de ser de fácil acesso, essa tecnologia também precisa ter seu conteúdo entendido (OLIVEIRA LMR, et al., 2018).

A diversidade de aplicativos de computação móvel é ampla, incluindo, por exemplo, suporte a diagnósticos médicos e de enfermagem, escolha da decisão certa, acesso remoto às informações do paciente por meio de registros médicos eletrônicos e acompanhamento após a alta, entre outros. Muitas soluções foram desenvolvidas para auxiliar em procedimentos médicos (NOGUEIRA JBS, et al., 2018).

As vantagens de usar aplicativos para dispositivos móveis podem ser caracterizadas em serem mais acessíveis, mais fáceis de operar, além de funcionaram como multitarefa e serem portáteis. Em relação à versatilidade, é preciso enfatizar que os dispositivos móveis fornecem ferramentas Web para colaboração interativa e acesso a aplicativos desenvolvidos especificamente para esses dispositivos. A utilização dos recursos móveis pode ser aplicada a diversos ramos dentro da área da saúde, como autogerenciamento, apoio diagnóstico, triagem e controle de doenças crônicas. Além disse apresenta-se como alternativa para a superação de obstáculos na assistência ao paciente, as tecnologias em saúde (eHealth) tem impacto significativo na eficiência, eficácia e equidade dos serviços, podendo otimizar recursos, reduzir custos e facilitar sua obtenção (MOSA AS, et al., 2012). 
Deve-se considerar que existem vários usos para aplicativos de monitoramento de saúde e qualidade de vida, variando de acordo com a disponibilidade dos sistemas operacionais, sendo relevante discutir a importância do uso de tecnologias para prevenção e promoção da saúde por ser uma modalidade de cuidado preconizada no país e por ter capacidade de realizar mudanças substanciais na lógica do cuidado ao propor práticas pautadas na responsabilidade compartilhada dos usuários pela sua saúde e redefinindo a saúde para avaliação (LEE SH, et al., 2016).

Observa-se que, no Brasil, a realização de estudos sobre aplicativos para dispositivos móveis voltados para a área da saúde ainda é escassa, mas apresenta evolução crescente e em constante expansão principalmente devido ao aumento da adesão e utilização de smartphones, tablets e computadores. No entanto, no campo internacional, o estudo de aplicações voltadas para saúde já é um ramo que rende diversas publicações e as ferramentas desenvolvidas se estendem a diversas áreas do cuidado a saúde. Enfermeiros, médicos e outros profissionais estão se apropriando de recursos tecnológicos buscando, resultados positivos para o paciente, família e equipe de saúde buscando melhorar a prática assistencial e a segurança do paciente (REAL FJ, et al., 2019).

No entanto, é importante entender que a atenção primária também deve desenvolver e integrar novas tecnologias de baixo custo para expandir o acesso ao serviço, reduzir custos e melhorar a eficácia do serviço. Exemplos dessa importância são iniciativas como a Política Nacional de Informação e Informática em Saúde, um de seus objetivos é melhorar os processos de trabalho, por meio da divulgação de informações específicas para as práticas profissionais e para a população em geral, e garantir o acesso aos serviços por meio da rede de atenção (GAVA M, et al., 2016).

A atenção básica desempenha um papel vital como primeiro ponto de contato com o sistema de saúde e ferramenta de atendimento contínuo, integral e coordenado. É especialmente importante para a solução dos problemas enfrentados pelos indivíduos com doenças crônicas e múltiplas comorbidades. A aplicação de tecnologia em saúde tem melhorado muito a vida dos pacientes, principalmente dos idosos, dos deficientes físicos e dos portadores de doenças crônicas. O uso adequado da tecnologia irá desencadear novas possibilidades para resolver os problemas urgentes de prestação e promoção de cuidados eficientes e eficazes (SILVA BMC, et al. 2015).

A saúde com suporte de tecnologia veio para ficar e continuará a evoluir, incluindo telemedicina, smartphones, aplicativos móveis, dispositivos domésticos e vestíveis e comunicações de vídeo online. Os médicos precisam ser bons no uso dessas tecnologias para promover a assistência médica de qualidade às pessoas. Isso exigirá treinamento clínico e desenvolvimento profissional, avanço tecnológico e políticas de reembolso revisadas. Dessa forma, a atenção primária evoluirá para redefinir seu papel no contexto dessas tecnologias emergentes (YOUNG HM e NESBIT TS, 2017).

Evidencia-se a importância de os aplicativos serem desenvolvidos cuidadosamente, com embasamento científico e técnicos apropriados e, posteriormente, testados os efeitos clínicos, em contextos diversos, envolvendo, também, populações com dificuldade de acesso à internet e tecnologias de informações. Ressalta-se, também a importância das evidências clínicas de alta qualidade para implicações terapêuticas confiáveis e de impacto na saúde (PAULA TR, et al., 2020).

\section{CONSIDERAÇÕES FINAIS}

A análise dos artigos nos mostrou que os aplicativos de tecnologia móvel desenvolvidos na geração de informações relacionados a Atenção Primária à Saúde concentram-se mais na área de atenção as condições crônicas principalmente para orientar profissionais e paciente sobre rastreamento e diagnóstico destas condições como também disponibilizam orientações sobre o tratamento. A participação brasileira na produção e publicação demonstradas nestas tecnologias foi discreta tendo em vista que o Brasil é potente em inovação e tecnologia. $O$ uso dos aplicativos teve significativa aprovação e aceitação no aspecto finalidades e satisfação dos usuários. Destaca-se a necessidade de disponibilizar estruturas e compartilhar redes através de apoio dos gestores de saúde na produção dessas ferramentas úteis a APS do país. 


\section{REFERÊNCIAS}

1. AHERN DK, et al. Feasibility of using patient-facing technology for the identification of COPD in primary care. Journal of Innovation in Health Informatics, 2016; 23(2):541-546.

2. BARRA CCD, et al. Métodos para desenvolvimento de aplicativos móveis em saúde: revisão integrativa da literatura. Texto \& Contexto Enfermagem, 2017; 26(4): e2260017.

3. BENNETT GG, et al. Effectiveness of an App and Provider Counseling for Obesity Treatment in Primary Care. American Journal of Preventive Medicine, 2018; 55(6): 777-786.

4. BONILLA C, et al. Use of electronic dietary assessment tools in primary care: An interdisciplinary perspective eHealth/ telehealth/ mobile health systems. BMC Medical Informatics and Decision Making, 2015; 15(1):1-13.

5. BRASIL. Manual do Sistema com Coleta de Dados Simplificada: CDS. Ministério da Saúde. Secretaria de Atenção à Saúde. e-SUS AB Atenção Básica, $2014 . \quad$ Disponível em: http://189.28.128.100/dab/docs/portaldab/documentos/manual_CDS_ESUS_1_3_0.pdf. Acessado em: 26 de set de 2021.

6. DA ROCHA, Fernanda Suzart et al. Uso de Apps para a promoção dos cuidāos à saúde. Anais do Seminário Tecnologias Aplicadas a Educação e Saúde, 2017.

7. DAHNE J, et al. Moodivate: A self-help behavioral activation mobile app for utilization in primary care-Development and clinical considerations. International Journal of Psychiatry in Medicine, 2017; 52(2): 160-175.

8. GAMA LN. TAVARES CMM. Desenvolvimento e avaliacão de aplicativo móvel na prevenção de riscos osteomusculares no trabalho de enfermagem. Texto contexto - enfermagem, 2019; 28(1): e20180214.

9. GAVA M, et al. Incorporação da tecnologia da informação na Atenção Básica do SUS no Nordeste do Brasil: expectativas e experiências. Ciência Saúde Coletiva, 2016; 21(3): 891-902.

10. GRANADO-FONT E, et al. Effectiveness of a Smartphone application and wearable device for weight loss in overweight or obese primary care patients: Protocol for a randomised controlled trial. BMC Public Health, 2015; 15(1): 2-7.

11. LEE, SH. et al. Effectiveness of mHealth interventions for maternal, newborn and child health in low-and middle-income countries: Systematic review and meta- analysis. Journal of global health, 2016, 6(1).

12. LÓPEZ MM, et al. Health App for iOS to Help in Diagnostic Decision in Ophthalmology to Primary Care Physicians. Journal of Medical Systems, 2017; 41(5).

13. LOUW C, et al. Smartphone-Based Hearing Screening at Primary Health Care Clinics. Ear and Hearing, 2017; 38(2): e93e100.

14. MARCANO BJS, et al. Comparison of self-administered survey questionnaire responses collected using mobile apps versus other methods. Cochrane Database Syst Revista, 2015; 27(7):MR000042.

15. MAULIK PK, et al. Increasing use of mental health services in remote areas using mobile technology: A pre- post evaluation of the SMART Mental Health proiect in rural India. Journal of Global Health, 2017; 7(1).

16. MEDHANYIE AA, et al. Quality of routine health data collected by health workers using smartphone at primary healthcare in Ethiopia. International Journal of Medical Informatics, 2017: 101: 9-14.

17. MENDES EV, et al. A construção social da atenção primária à saúde. $2^{\underline{a}}$ ed. Brasília: Conselho Nacional de Secretários de Saúde (CONASS), 2019; 192p.

18. MILLER DP, et al. Effect of a digital health intervention on receipt of colorectal cancer screening in vulnerable patients a randomized controlled trial. Annals of Internal Medicine, 2018; 168(8): 550-557.

19. MILLS K, et al. Protocol for the melatools skin self-monitoring trial: a phase II randomised controlled trial of an intervention for primary care patients at hiaher risk of melanoma. BMJ Open, 2017; 7:e017934.

20. MOSA AS, et al. A systematic review of healthcare applications for smartphones. BMC, 2012; 12(67):14-21.

21. NOGUEIRA JBS, et al. Avaliação de utilidade e acurácia de aplicativo móvel no planejamento de artroplastias totais do joelho. Revista brasileira de ortopedia, 2018; 53(2):142-150.

22. OLIVEIRA ARF, ALENCAR MSM. O uso de aplicativos de saúde para dispositivos móveis como fontes de informação e educacão em saúde. Revista Diqital de Biblioteconomia e Ciência da Informacão, 2017; 15 (1), 234-245.

23. OLIVEIRA LMR, et al. Tecnologia mHealth na prevenção e no controle de obesidade na perspectiva do letramento em saúde: Lisa Obesidade. Saúde debate, 2018; 42(118): 714-723.

24. PACHECO KCF, et al. A construção de objeto de aprendizagem sobre doenças transmissíveis para agentes comunitários de saúde. Revista Gaúcha de Enfermagem, 2018; 38(4): e20170073.

25. PAULA TR, et al. Efetividade de aplicativos móveis para mudanças comportamentais em saúde: revisão sistemática. Revista Rene, 2010; 21: e43845.

26. PEREIRA FGF, et al. Construção de um aplicativo digital para o ensino de sinais vitais. Revista Gaúcha de Enfermagem, 2016;37(2): 1-7.

27. PEREIRA IM, et al. Tecnologia móvel para coleta de dados de pesquisas em saúde. ACTA Paulista de Enfermagem, 2017; 30(5): 479-488.

28. POSSEMATO K. Development and refinement of a clinician intervention to facilitate primary care patient use of the PTSD Coach app. Translational Behavioral Medicine, 2017; 7(1): 116-126.

29. PROESMANS T, et al. Mobile phone-based use of the photoplethysmography technique to detect atrial fibrillation in primary care: Diagnostic accuracy study of the fibricheck app. JMIR MHealth and UHealth, 2019; 7(3): 1-11.

30. REAL FJ, et al. Dose Matters: A Smartphone Application to Improve Asthma Control Among Patients at an Urban Pediatric Primary Care Clinic. Games for Health Journal, 2019; 8(5): 357-365.

31. RECIO-RODRIGUEZ JI, et al. The effectiveness of a smartphone application on modifying the intakes of macro and micronutrients in primary care: A randomized controlled trial. the EVIDENT II study. Nutrients, 2018; 10(10): 1-16.

32. ROBERTS S, et al. Using technology to engage hospitalised patients in their care: a realist review. BMC Health Serv. Res. 2017; 17: 388.

33. SENEVIRATNE MG, et al. HealthNavigator: A mobile application for chronic disease screening and linkage to services at an urban Primary Health Network. Australian Journal of Primary Health, 2018; 24(2): 116-122.

34. SILVA AB. Adaptação transcultural do aplicativo Zero Mothers Die para dispositivos móveis no Brasil: contribuicões para a saúde digital com abordagem do cuidado centrado na e-gestante. Revista Brasileira de Saúde Materno Infantil, 2019, 19(4): 763-775.

35. SILVA BMC, et al. Mobile-health: A review of current state in 2015. Journal of biomedical informatics, 2015; 56: $265-272$.

36. TETAMANTI JMD, et al. Desarrollo de un sistema georreferenciado para la gestión, movilidad y monitoreo de atención primaria de la salud comunitaria. Salud Colectiva, 2018; 14(1): 121-137.

37. TIBES CMS, et al. Mobile applications developed for the health sector in Brazil: an integrative literature review. Reme: Revista Mineira de Enfermagem, 2014; 18(2): 471-478.

38. TSOH JY, et al. Interactive mobile doctor (IMD) to promote patient-provider discussion on tobacco use among asian American patients in primary care: A pilot study. Journal of the American Board of Family Medicine, 2018; 31(6): 869-880.

39. YOUNG HM, NESBITT TS. Increasing the Capacity of Primary Care Through Enabling Technology. Journal of General Internal Medicine, 2017; 32(4): 398 - 403. 